\title{
Editorial on Carcinoma Prostate
}

\author{
Nayab Mustansar* \\ Consultant Nuclear Physician, Pakistan Atomic Energy, Islamabad, Pakistan \\ *Corresponding author: Nayab Mustansar, Consultant Nuclear Physician, Pakistan Atomic Energy, Islamabad, Pakistan. \\ To Cite This Article: Nayab Mustansar. Editorial on Carcinoma Prostate. Am J Biomed Sci \& Res. 2019 - 4(1). AJBSR.MS.ID.000745. \\ DOI: 10.34297/AJBSR.2019.04.000745
}

Received: July 01, 2018 | Published: July 12, 2019

\section{Editorial}

When healthy and normal cells in the body change and grow out of control it forms a cancer. There are different kinds of cancers. Most common are the breast Cancer, thyroid cancer and prostate cancer. Breast cancer and prostate cancer spread to bones most commonly. A cancer can be a benign cancer or a malignant cancer. A malignant tumour has a metastatic spread that is it spread to other organs whereas a benign cancer can grow but cannot spread [1].

Prostate cancer is one the unusual yet very common cancer affecting most of the population. It is different from rest of the cancers as it doesn't spread quickly to other parts of body. Mostly the prostate cancer is slowly growing and doesn't cause any obvious sign and symptoms. The usual symptoms are frequent and painful micturation, burning micturation, blood in urine and seminal fluid, weak and interrupted urine flow and new onset of erectile dysfunction [2]. Prostate cancer is somewhat unusual when compared with other types of cancer. This is because many prostate tumours do not spread quickly to other parts of the body. Some prostate cancers grow very slowly and may not cause symptoms or problems for years.

Usually the early stage of prostate cancer has no signs and symptoms. It is usually detected via DRE or PSA test. If it suggests of having a Carcinoma prostate than further testing is carried out [3]. A significant Research is being carried out for early detection of prostate cancer and its linkage between the nutrition and lifestyle factors. New genomic tests have been introduced by researchers for its proper management [4].
Till date there are a lot of treatment options which include Radiotherapy, chemotherapy and anti cancer drugs. For advanced metastatic cancer especially to bones a lot of quantitative parameters have been introduced, which include \%PAB (Positive area on bone scans), \%BSI (Bone scan index), BLS (bone Lesion scoring) and EOD (Extent of Disease). These methods help in quantifying the tumour burden on bone scans which ultimately helps in the modification of treatment plan [5]

\section{References}

1. Folkman J, Cotran R (1976) Relation of vascular proliferation to tumor growth. Int Rev Exp Pathol 16: 207-248.

2. Weidner N, Semple JP, Welch WR, Folkman J (1991) Tumor angiogenesis and metastasis. Correlation in invasive breast carcinoma. N Engl J Med 324(1): 1-8.

3. Arakawa A, Soh S, Chakraborty S, Wheeler TM (1997) Prognostic significance of angiogenesis in clinically localized prostate cancer (staining for factor VIII-related antigen and CD34 antigen). Prostate Cancer Prostatic Diseases 1(1): 32-38.

4. Silberman MA, Partin AW, Veltri RW, Epstein JI (1997) Tumor angiogenesis correlates with progression after radical prostatectomy but not with pathologic stage in Gleason sum 5 to 7 adenocarcinoma of the prostate. Cancer 79(4): 772-779.

5. Nakajima K, Nakajima Y, Horikoshi H, Ueno M, Wakabayashi $H$, et al. (2013) Enhanced diagnostic accuracy for quantitative bone scan using an artificial neural network system: a Japanese multi-center database project. EJNMMI Res 3(1): 83. 